Direktor der Klinik und Poliklinik für Frauenheilkunde und Geburtshilfe, Universitätsklinikum Köln (AöR), Kerpener Str. 34, 50931 Köln

\title{
Weniger ist mehr
}

D iese einfache Formel fasst prägnant die derzeitige Entwicklung in der gynäkologischen Onkologie zusammen, was auch auf der diesjährigen Tagung der American Society of Clinical Oncology (ASCO) eindrucksvoll bestätigt wurde. Dass beim frühen Zervixkarzinom ein organerhaltendes Vorgehen mit Konisation und Sentinel-Node-Biopsie ausreicht, ist bereits in den seit 2014 gültigen S3-Leitlinien festgelegt worden. Dass man jetzt aber auch das klassische Zervixkarzinom durch eine einfache Hysterektomie oder sogar eine Konisation mit gleicher onkologischer Sicherheit operieren kann, ist neu. Noch eindrucksvoller ist die Entwicklung beim Ovarialkarzinom: Die bislang routinemäßig durchgeführte Lymphonodektomie beim R0-operierten Ovarialkarzinom ohne klinisch vergrößerte Lymphknoten wird zukünftig wahrscheinlich aufgrund der Daten der LION(Lymphadenectomy In Ovarian Neoplasms)Studie obsolet. Die Entwicklung in der gynäkologischen Onkologie nähert sich mit circa 20-jähriger Verzögerung der operativen Therapie des Mammakarzinoms an. Ähnlich wie beim Mammakarzinom reicht offenbar auch bei den gynäkologischen Karzinomen die Resektion in sano aus. Mehr braucht es anscheinend nicht.

Dies zeigt in leider enttäuschender Weise auch die Entwicklung beim Ovarialkarzinom. Betrachtet man die Daten des Tumorregisters München oder des Krebsregisters NRW, dann hat sich bei der Behandlung des Ovarialkarzinoms seit fast 30 Jahren nichts geändert. Die Überlebenswahrscheinlichkeit einer Patientin mit Ovarialkarzinom ist im Jahr 2017 nicht besser als im Jahr 1988, trotz der Einführung neuer Medikamente wie der Taxane und des Bevacizumab in der adjuvanten Therapie, trotz immer radikaleren operativen Vorgehens, trotz Qua- litätssicherung und Etablierung neuer Strukturen wie der Gynäkologischen Krebszentren. Besonders enttäuschend ist dies für uns Operateure. Mit großem Einsatz bemühen sich alle gynäkologischen Onkologen um eine möglichst hohe Rate an R0-resezierten Patientinnen mit Ovarialkarzinom. Darmresektion, Leberteilresektion, Milzentfernung, Deperitonealisierung usw. haben zwar den Anteil der komplett R0-operierten Patientinnen erhöht, aber trotzdem überleben nur maximal $40 \%$ von ihnen.

Entscheidend bleibt in der Onkologie offenbar die Tumorbiologie, die sich auch mit dem Messer nicht beeinflussen lässt. Dieser Tatsache trägt auch die Entwicklung der medikamentösen Therapie in der gynäkologischen Onkologie Rechnung. Die verschiedenen Optionen einer zielgerichteten Therapie sind in unserem Fachgebiet zwar derzeit noch im Regelfall in Kombination mit einer Chemotherapie oder als Erhaltungstherapie nach Chemotherapie wirksam, es besteht jedoch die berechtigte Hoffnung, dass auch hier, ähnlich wie bei den hämatologischen Erkrankungen oder beim Melanom, neue Möglichkeiten der zielgerichteten Therapie auf den Markt kommen werden, die exakt den molekularen Defekt angreifen, der individuell für jeden Patienten im Rahmen eines Next Generation Sequencing identifiziert wurde. Die individualisierte zielgerichtete Therapie ist die große Hoffnung in der gynäkologischen Onkologie für die $\mathrm{Zu}$ kunft, alles andere, das zeigt die Entwicklung beim Ovarialkarzinom, reicht für einen Durchbruch nicht aus.

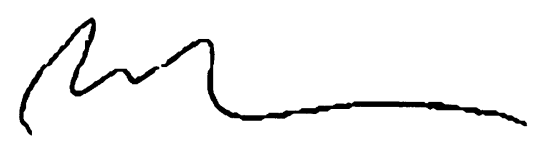

